\title{
Automatic exposure compensation and subjective image enhancement in the radiographic diagnosis of caries
}

\author{
Neiandro Santos GALVÃO(a) iD \\ Eduarda Helena Leandro \\ NASCIMENTO(a) \\ Hugo GAÊTA-ARAUJO(a) \\ Deborah Queiroz FREITAS(a) ID \\ Francisco HAITER-NETO(a) ID \\ Matheus Lima OLIVEIRA(a) iD \\ (a) Universidade Estadual de Campinas \\ - Unicamp, Piracicaba Dental School, \\ Department of Oral Diagnosis, Piracicaba, \\ SP, Brazil.
}

Declaration of Interests: The authors certify that they have no commercial or associative interest that represents a conflict of interest in connection with the manuscript.

Corresponding Author:

Matheus Lima Oliveira.

E-mail:matheuso@unicamp.br

htpps://doi.org/10.1590/1807-3107bor-2020.vol34.0082

Submitted: December 2, 2019

Accepted for publication: April 23, 2020

Last revision: June 2, 2020
Abstract: This study aims to assess the influence of high-density material on the radiographic diagnosis of proximal caries in digital systems with automatic exposure compensation, and to evaluate the effect of subjective adjustment of brightness and contrast to undertake this diagnostic task. Twenty bitewing radiographs of forty posterior human teeth with non-cavitated carious lesions, confirmed by micro-CT, were obtained with two digital systems. A porcelain-fused-to-metal crown attached to a titanium implant was inserted into the exposed area, and all the radiographs were repeated. Five radiologists assessed the radiographs and diagnosed proximal carious lesions. Afterwards, the observers were asked to adjust image brightness and contrast, based on their subjective perception, and to reassess the images. Thirty percent of each experimental group was reassessed to test intraobserver reproducibility, totaling 208 images per observer. Intraobserver and interobserver agreements ranged from fair to substantial. Sensitivity, specificity, predictive values, and area under the ROC curve were calculated and compared for each radiographic system, using ANOVA $(\alpha=0.05)$. Overall, presence of high-density material and adjustment of brightness and contrast did not significantly influence the radiographic diagnosis of proximal caries ( $p \geq 0.05$ ). Regarding Digora Optime, adjustment of brightness and contrast significantly increased $(p<0.05)$ the diagnostic accuracy of proximal carious lesions in the presence of high-density material. In conclusion, the presence of high-density material in the X-rayed region does not influence radiographic diagnosis of proximal caries. However, when it is present in the $\mathrm{X}$-rayed area, subjective adjustment of brightness and contrast is recommended for use with the Digora Optime digital system.

Keywords: Radiography, Dental, Digital; Dental Caries; Diagnostic Imaging; Radiographic Image Enhancement.

\section{Introduction}

Digital intraoral radiographic systems are widely used in many areas of dentistry. For this reason, digital image receptors have been constantly updated to improve image quality, and possibly increase diagnostic accuracy. ${ }^{1}$ Accordingly, in addition to image post-processing tools allowing the observer to subjectively adjust features such as brightness 
and contrast, some digital systems also pre-process the image before it is displayed on the monitor. In this respect, automatic exposure compensation (AEC) is an observer-independent pre-processing tool that adjusts the grayscale range based on the amount of X-ray reaching the image receptor. ${ }^{2,3}$ This prevents important image degradation when exposure factors are not optimum, and has a positive effect on patient dose control. ${ }^{4,5}$

The existing scientific literature is rather limited concerning AEC; however, digital systems having AEC have demonstrated greater accuracy in the radiographic diagnosis of carious lesions at inadequate exposure factors. ${ }^{3}$ Importantly, in addition to the energy factors that can be adjusted on the X-ray machine, such as exposure time, milliamperage and kilovoltage, the physical properties of the X-rayed object can also affect the amount of radiation reaching the image receptor. Recent in-vitro studies ${ }^{2,6}$ have shown that the presence of high-density materials affect the gray values of digital radiographic images; furthermore, radiographic images of specific digital systems obtained at constant exposure factors tend to be much denser (darker) when the AEC is triggered by metallic materials. From a clinical perspective, dental practitioners should be aware that any factor affecting the final image quality must be tested to determine how well it has performed several different diagnostic tasks, before it can be used in the clinical routine, regardless of the manufacturer's claim that such a tool was developed to improve the diagnosis.

Considering that numerous metallic materials can be observed in the oral cavity (e.g. dental implants, metallic restorations, and prosthesis) and that the diagnosis of carious lesions is based on the detection of subtle differences in shades of gray, it is important to determine the indirect influence of high-density material, by activating the AEC to perform this highly frequent diagnostic task in dentistry. To the best of the authors' knowledge, this is the first study focusing on the clinical impact of AEC when high-density materials are present in the exposed area. Thus, this study aimed: a) to assess the influence of high-density material on the radiographic diagnosis of proximal caries, in digital systems with $\mathrm{AEC}$, and b) to evaluate the effect of the subjective adjustment of brightness and contrast to undertake this diagnostic task. The null hypothesis claims that the presence of high-density material in the exposed area, and the subjective adjustment of brightness and contrast, did not influence the diagnosis of carious lesions in the digital radiographic systems having AEC studied herein.

\section{Methodology}

This study was approved by the institutional human research ethics committee (protocol \#65745217.1.0000.5418).

\section{Sample selection}

Forty posterior human teeth ( 20 premolars and 20 molars) were selected from a sample made available by a private dental practitioner, who extracted them for reasons unrelated to the present study. All the teeth were previously subjected to professional prophylaxis and to the inclusion criteria, considered suspected or distinct proximal white spot lesions on at least one proximal surface (scores 1 or 2 according to ICDAS-International Caries Detection and Assessment System). ${ }^{7}$ Teeth with cavitated lesions, restorations, and anomalous anatomy were not included in the sample.

\section{Reference standard}

The presence and extension of the carious lesions was confirmed by scanning all the teeth with the Skyscan 1174 micro-computed tomography unit (Bruker Corp., Kontich, Belgium) at 50 kV, $800 \mu \mathrm{As}$, aluminum filter of $0.5 \mathrm{~mm}$, pixel size of $15 \mu \mathrm{m}, 1$ frame, rotation step of $0.3^{\circ}$, rotation of $180^{\circ}, 617$ basis images and scanning time of 57 minutes. Two experienced oral radiologists jointly evaluated all the images and determined the reference standard for carious lesions, by consensus. The sample of 80 proximal surfaces (40 teeth $X 2$ proximal surfaces) was diagnosed with 27 sound surfaces, 35 surfaces with carious lesions restricted to the outer half of the enamel, and 18 surfaces with carious lesions reaching the dentin-enamel junction (Figure 1). 

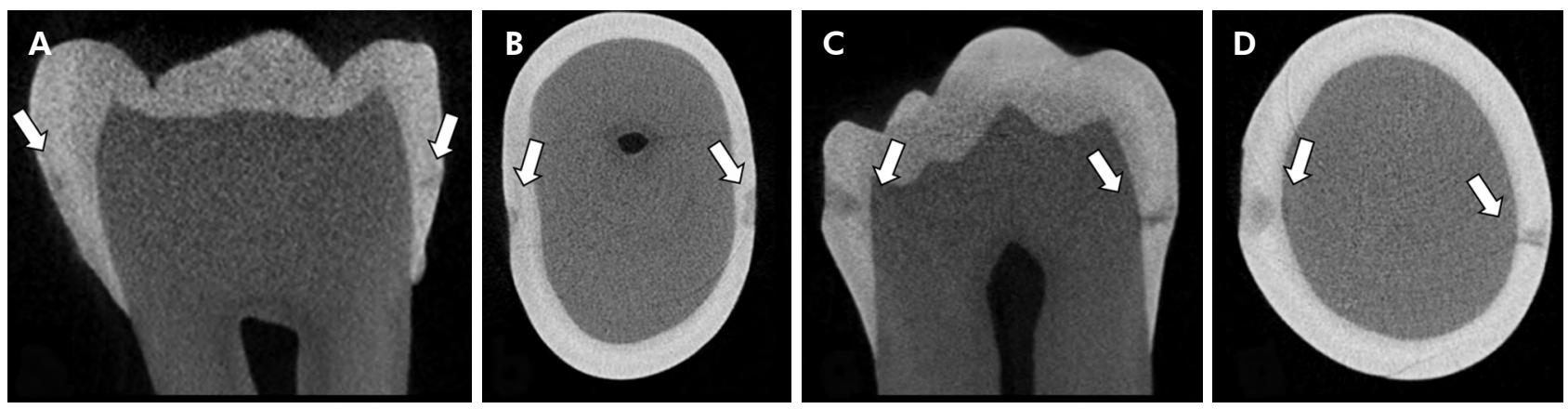

Figure 1. Micro-computed tomography images showing proximal carious lesions (white arrows) confined to the outer surface of enamel $(A$ and $B)$ and reaching the dentin-enamel junction ( $C$ and $D)$.

\section{Radiographic phantom preparation}

The forty selected teeth were grouped into twenty pairs, and inserted into radiographic phantoms composed of six extra (non-test) teeth - one canine, two premolars, and three molars - fixed in two plaster blocks mixed with powdered rice in a proportion of 1:1 to simulate the radiographic appearance of alveolar bone. ${ }^{8}$ As shown in Figure 2A, the teeth were arranged in such a way as to simulate a clinical condition. One of the non-test teeth could easily be removed for later replacement with metallic material (Figure 2B).

\section{Radiographic image acquisition}

The radiographic phantom (Figure 2A) was X-rayed using the Focus unit (Instrumentarium, Tuusula, Finland) at $70 \mathrm{kVp}, 7 \mathrm{~mA}$, exposure time of $0.16 \mathrm{~s}$ and a focus-image receptor distance of $40 \mathrm{~cm}$, using the interproximal technique. Two digital radiographic imaging systems were used, both having AEC: the Digora Toto (Soredex, Tuusula, Finland), size 2 CMOS-sensor with bit depth of 12 and spatial resolution of $26.3 \mathrm{lp} / \mathrm{mm}$, and the Digora Optime (Soredex, Tuusula, Finland), size 2 phosphor plate, with bit depth of 14 and spatial resolution of $14.3 \mathrm{lp} / \mathrm{mm}$. Afterwards, a non-test tooth was replaced with a set composed of a titanium implant and a porcelain-fused-to-metal crown (Figure 2B). Radiographic acquisitions were repeated using the same exposure parameters, and generated a total of 80 images (20 pairs of teeth $\times 2$ digital systems $\times 2$ high-density material conditions with and without a high-density material $+30 \%$ reassessment). The high-density material was inserted to induce AEC activation.

\section{A}

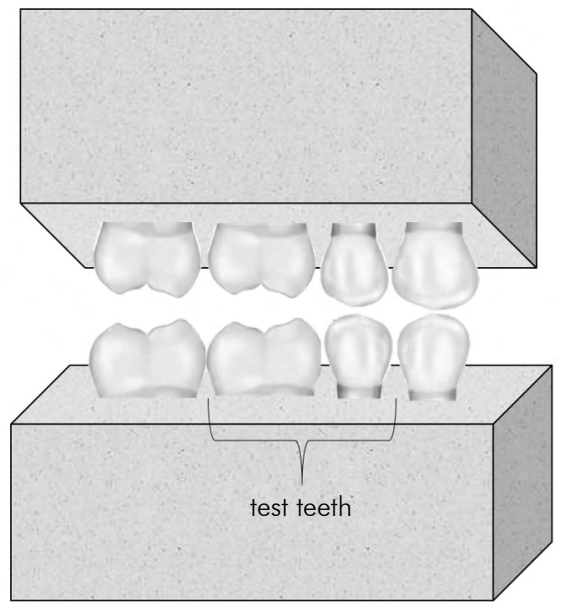

B

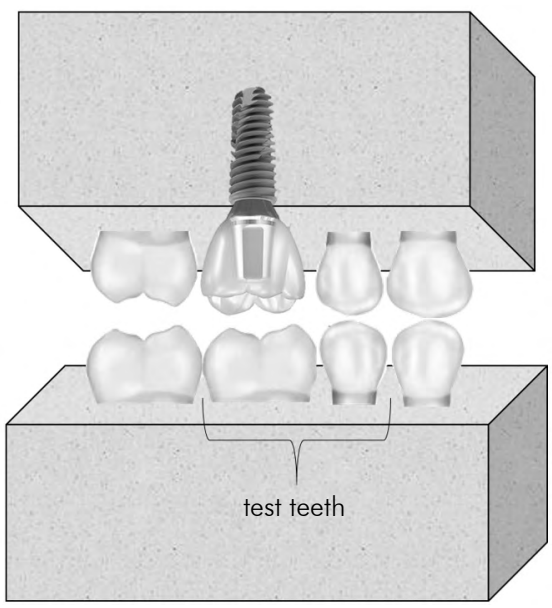

Figure 2. Schematic drawing of the radiographic phantoms indicating the pair of test teeth in the absence $(A)$ and presence (B) of high-density material. 
A platform was designed to stabilize the phantom and hold the image receptor, so that the image acquisition could be standardized and the same geometrical setting could be maintained to apply the interproximal technique. A $2.5 \mathrm{~cm}$-thick acrylic plate was placed between the X-ray source and the phantom to simulate soft-tissue attenuation. All images were exported as TIFF files with their original spatial and contrast resolutions: Digora Toto, at 12 bits and $26.3 \mathrm{lp} / \mathrm{mm}$, and Digora Optime, at 14 bits and $14.3 \mathrm{lp} / \mathrm{mm}$ (Figure 3).

\section{Image assessment}

All images were encoded and randomized using a free online service available at www.random. org. Five oral radiologists, with at least five years of experience in diagnosing proximal caries, assessed the images independently using the JPEGView, a public domain software program, viewed on a 24.1-inch, 1920x1200-pixel LCD monitor (Barco N.V., Courtray, Belgium), in a calm and low-light environment. At this time, no subjective image adjustment was allowed. The observers were asked to score both proximal surfaces of each test tooth on a 5-point scale, according to the presence of carious lesions: (1) absent, (2) probably absent, (3) uncertain, (4) probably present and (5) present. After one month, the observers were asked to reassess all the radiographic images in a new randomized order, and were advised to adjust only image brightness and contrast, based on their subjective perception. After an additional one-month interval, $30 \%$ of each experimental group (with and without high-density material, and with and without adjustment of brightness and contrast) was reassessed to test intraobserver reproducibility.
All evaluations resulted in a total of 208 images per observer ( 80 images $\times 2$ adjustment conditions: with and without adjustment). The intraobserver reproducibility was tested by reassessing $30 \%$ of the images. The authors assessed interobserver agreement by comparing the outcomes of the evaluation of all 5 observers, compared in pairs, as such: observer 1 was compared with 2, 34 and 5; observer 2 was compared with 3,4 , and 5 ; observer 3 was compared with 4 and 5, and observer 4 was compared with 5 .

\section{Statistical analysis}

The data were analyzed using SPSS version 22.0 software (IMB, Armonk, New York, USA). The Shapiro-Wilk test was used to assess the distribution of the sample, which proved to be normal ( $p>0.05)$. Intra- and interobserver agreement was calculated using the weighted Kappa, and was interpreted based on Landis \& Koch', according to which a range between 0.00 and 0.20 indicates slight agreement, between 0.21 and 0.40 indicates fair agreement, between 0.41 and 0.60 indicates moderate agreement, between 0.61 and 0.80 indicates substantial agreement, and between 0.81 and 1.00 indicates excellent or almost perfect agreement. The scores assigned by the observers were used to calculate the area under the receiver operating characteristic curve (AUC). Sensitivity, specificity, positive predictive value (PPV) and negative predictive value (NPV) were calculated by dichotomizing the data, considering scores 1,2 and 3 as absence of caries, and scores 4 and 5 as presence of caries. All diagnostic values were calculated for each observer individually, and were expressed as mean values and standard deviation. The groups were compared using the ANOVA with
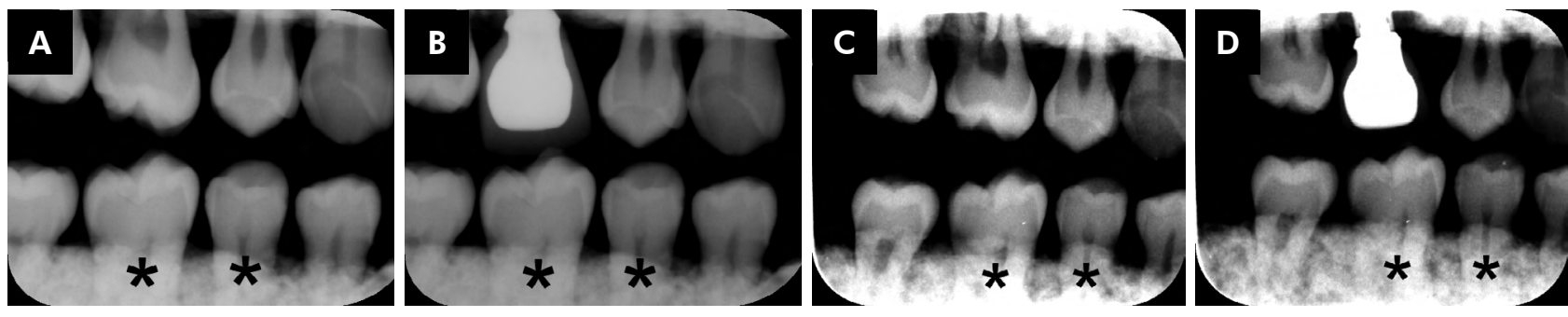

Figure 3. Bitewing digital radiographs obtained with the Digora Toto (A and $B$ ) and Digora Optime (C and D), in the absence ( $A$ and $C$ ) and presence ( $B$ and $D$ ) of high-density material. Asterisks indicate the test teeth. 
post-hoc Tukey test to check the influence of the factors studied. The significance level was set at $5 \%$ $(\alpha=0.05)$. The power of the test was 0.8 , based on the mean minimum differences among the groups, and the mean standard deviation (Biostat software, v. 5.3, Instituto de Desenvolvimento Sustentável Mamirauá, Tefé, Brazil).

\section{Results}

The intraobserver agreement for both digital radiographic systems ranged from moderate (0.541) to substantial (0.721), and the interobserver agreement ranged from fair $(0.228)$ to moderate $(0.426)$ (Table 1$)$.

Table 2 shows that the presence of high-density material did not significantly affect the radiographic diagnosis of proximal carious lesions $(\mathrm{p} \geq 0.05)$ for either digital system. The adjustment of brightness and contrast on the Digora Optime significantly increased $(p<0.05)$ the diagnostic accuracy of proximal carious lesions in the presence of high-density material. In

Table 1. Kappa values for intraobserver (in bold) and interobserver agreements (among the 5 observers).

\begin{tabular}{lccccc}
\hline Observer & 1 & 2 & 3 & 4 & 5 \\
\hline 1 & $\mathbf{0 . 5 7 6}$ & 0.278 & 0.426 & 0.418 & 0.371 \\
2 & & $\mathbf{0 . 7 2 1}$ & 0.352 & 0.422 & 0.228 \\
3 & & & $\mathbf{0 . 5 5 4}$ & 0.398 & 0.283 \\
4 & & & & $\mathbf{0 . 6 3 7}$ & 0.318 \\
5 & & & & & $\mathbf{0 . 5 4 1}$ \\
\hline
\end{tabular}

most cases, specificity was greater than sensitivity, and PPV was greater than NPV in all cases. No significant difference was observed in the values of specificity, sensitivity, PPV and NPV, among the experimental conditions of both digital radiographic systems $(p \geq 0.05)$.

\section{Discussion}

Limited information is currently available on the effectiveness of AEC in digital intraoral radiographic imaging systems for different diagnostic tasks. To the best of the authors' knowledge, this is the first study to evaluate the influence of high-density material on the radiographic diagnosis of proximal caries in digital systems with AEC, as well as the effect of subjective adjustment of brightness and contrast. Interestingly, our outcomes reveal that the presence of high-density material in the X-rayed region did not influence the diagnosis of proximal carious lesions by the two digital radiographic systems tested: Digora Toto and Digora Optime. However, the adjustment of brightness and contrast increased the diagnostic accuracy of the Digora Optime, in the presence of high-density material.

In-vitro studies conducted in 1996 assessed the AEC-also referred to as equalization-of two digital imaging systems, namely, Computed Dental Radiography (Schick Technologies Inc., Long Island City, USA) ${ }^{4}$ and RVG-4 (Trophy Radiologie, Vincennes, France), ${ }^{5}$ and showed that it affects image contrast and

Table 2. Mean values (standard deviation) of the area under the ROC curve (AUC), sensitivity, specificity, positive predictive value (PPV) and negative predictive value (NPV), in the radiographic diagnosis of proximal caries using two digital systems, with and without (w/o) a high-density material and adjustment of brightness and contrast.

\begin{tabular}{|c|c|c|c|c|c|c|c|}
\hline $\begin{array}{l}\text { Radiographic } \\
\text { system }\end{array}$ & $\begin{array}{l}\text { High-density } \\
\text { material }\end{array}$ & $\begin{array}{c}\text { Adjustment of } \\
\text { brightness and contrast }\end{array}$ & AUC & Sensitivity & Specificity & PPV & NPV \\
\hline \multirow{4}{*}{ Digora Toto } & \multirow{2}{*}{$w / o$} & w/o & $0.43(0.02)^{a}$ & $0.31(0.11)^{a}$ & $0.58(0.10)^{a}$ & $0.50(0.05)^{a}$ & $0.35(0.01)^{\circ}$ \\
\hline & & with & $0.46(0.02)^{a}$ & $0.37(0.16)^{a}$ & $0.57(0.10)^{a}$ & $0.54(0.07)^{a}$ & $0.38(0.02)^{\circ}$ \\
\hline & \multirow{2}{*}{ with } & $w / o$ & $0.44(0.02)^{a}$ & $0.31(0.10)^{a}$ & $0.59(0.07)^{a}$ & $0.52(0.05)^{a}$ & $0.37(0.01)^{\circ}$ \\
\hline & & with & $0.44(0.06)^{a}$ & $0.35(0.16)^{a}$ & $0.58(0.09)^{a}$ & $0.53(0.09)^{a}$ & $0.38(0.05)^{\circ}$ \\
\hline \multirow{4}{*}{ Digora Optime } & \multirow{2}{*}{ w/o } & $w / o$ & $0.49(0.08)^{a b}$ & $0.49(0.18)^{a}$ & $0.50(0.13)^{a}$ & $0.56(0.03)^{a}$ & $0.44(0.15)^{\circ}$ \\
\hline & & with & $0.48(0.02)^{a b}$ & $0.55(0.19)^{a}$ & $0.44(0.21)^{a}$ & $0.60(0.03)^{a}$ & $0.39(0.03)^{\circ}$ \\
\hline & \multirow{2}{*}{ with } & $w / o$ & $0.42(0.03)^{b}$ & $0.32(0.15)^{a}$ & $0.58(0.11)^{a}$ & $0.58(0.03)^{a}$ & $0.38(0.04)^{\circ}$ \\
\hline & & with & $0.51(0.04)^{a}$ & $0.49(0.22)^{a}$ & $0.53(0.23)^{a}$ & $0.61(0.04)^{a}$ & $0.41(0.05)^{\circ}$ \\
\hline
\end{tabular}

Different letters after the values indicate statistical significant difference $(p \leq 0.05)$ in the same column within each radiographic system, according to one-way ANOVA test. 
signal-to-noise ratio when sub-optimal exposures are used. Recently, focusing on the physical properties of the X-rayed object rather than on the exposure time, an in-vitro study ${ }^{2}$ revealed that the presence of high-density material significantly affected the gray values of the radiographic image. However, this was a technical methodology with no clinical simulation, in which the gray values of an aluminum step-wedge were assessed in the presence and absence of a lead plate. Similarly, in another in-vitro study, AEC altered the dental tissue-equivalent gray values in the presence of a material equivalent to a titanium implant. ${ }^{6}$ Nevertheless, the present study revealed that the AEC-related gray value variation of the radiographic image was not sufficient to affect the diagnostic accuracy of proximal carious lesions, which may be attributed to the simulation of a clinical condition with much more attenuating structures.

The radiographic detection of proximal caries has been studied extensively in the literature, ${ }^{1,10,11,12,13}$ however, the objectives addressed have focused mostly on the comparison between analogue and digital systems, ${ }^{1,10,12,13}$ and the use of post-processing methods. ${ }^{10,14,15,16}$ Overall, these studies have not shown any significant differences between conventional and digital methods, even when the image is post-processed. A previous study ${ }^{3}$ investigated the effect of AEC on the diagnosis of proximal caries using radiographic examinations at different exposure times, and concluded that digital systems having AEC increased the diagnostic accuracy of proximal caries in cases of both under- and overexposure. However, unlike our methodology, it did not consider the influence of high-density materials on the $X$-rayed area.

In the present study, the intraobserver agreement ranged from moderate to good, which reveals the consistency of the observers in their responses at different times. Although the interobserver agreement was lower (from reasonable to moderate), it could be expected, based on the subjectivity of radiographic evaluations, especially those involving the detection of very subtle changes in the image.$^{10}$ Regarding the low diagnostic values (AUC, specificity, sensitivity, PPV, and NPV) found in the present study, the authors believe that this is mostly because the sample was exclusively and intentionally composed of non-cavitated carious lesions, evidently making its radiographic diagnosis even more difficult. ${ }^{10,11}$ Thus, the sample was designed specifically to assess how AEC can affect a challenging diagnostic task. Interestingly, the low values were similar to those obtained in previous studies. ${ }^{11,12,14}$

Comparing the two radiographic systems studied, both presented similar results and were not influenced by the presence of the high-density material. The only significant difference was found in the accuracy values of the Digora Optime system, in which the brightness and contrast adjustments improved the performance of the evaluators in the presence of the high-density material. According to Nascimento et al., ${ }^{10}$ these adjustments do not influence the diagnosis of proximal carious lesions within an acceptable range of image quality. However, these authors did not consider the presence of high-density materials. ${ }^{10}$ Therefore, we believe that the adjustments of brightness and contrast studied herein compensated the AEC-related modifications in the Digora Optime.

The present methodology made use of the bitewing radiographic technique, because it is the most appropriate imaging modality for the diagnosis of proximal caries. ${ }^{16}$ Additionally, considering that the real action of the AEC is not disclosed by manufactures, and that previous studies showed distinctive effects of AEC for different digital systems, ${ }^{2,3}$ the authors decided to include only digital radiographic imaging systems manufactured by the same company. Importantly, the technology of the image receptor is different from that of the two digital systems, namely, the sensor-based receptor of the Digora Toto, and the phosphor plate-based receptor of the Digora Optime. Although many differences can be listed between the two digital systems in terms of composition, image formation, and physical aspects, both have very high spatial and contrast resolutions: those of Digora Toto are $26.3 \mathrm{lp} / \mathrm{mm}$ and 12 bits, and those of Digora Optime, $14.3 \mathrm{lp} / \mathrm{mm}$ and 14 bits, these being fundamental characteristics needed for diagnostic purposes. Furthermore, not only is a good quality digital system needed as a fundamental requirement for diagnostic purposes, but the geometrical principles of the radiographic technique must be strictly observed. Nonobservance 
could represent a relatively negative aspect of sensor-based digital systems, because of the greater thickness of their image receptor - a feature that can make the positioning more difficult for a less experienced professional. Use of micro-computed tomography as the reference standard for detecting carious lesions is relatively recent, but has presented substantial results. ${ }^{17,18,19}$ The great advantage of micro-computed tomography is its non-destructive feature, compared to histological methods.

Digital radiographic systems present many characteristics that can influence diagnostic accuracy, such as contrast and spatial resolutions, dynamic scaling and integrated image enhancement software. ${ }^{1,10,14}$ Therefore, the comparison of results obtained from different radiographic systems must be made respecting the characteristics of each system, and cannot be generalized to all of them. ${ }^{20}$ This explains why digital radiographic systems were not compared directly in our study. In addition, we believe that the location and amount of high-density material in the radiograph did not influence our results, because the action of the AEC seems to be based mainly on the highest and lowest pixel values of the image, and not on the quantity and location of these pixels. ${ }^{3,6}$ Furthermore, because this was an ex-vivo study, it had the relevant limitation of lacking clinical signs (sensitivity and/or pain) in the diagnostic process. Conversely, the present methodology enabled greater control of the variables, standardization of the radiographic acquisitions and comparison of the diagnosis with a reference standard, factors that contributed toward obtaining sounder results. Further studies evaluating additional diagnostic tasks and a wider range of digital radiographic systems with AEC are recommended.

\section{Conclusion}

The presence of high-density material in the $\mathrm{X}$-rayed region did not influence the radiographic diagnosis of proximal carious lesions. However, in such cases, subjective adjustment of brightness and contrast is recommended when using the Digora Optime digital system.

\section{Acknowledgments}

This study was financed in part by the Coordenação de Aperfeiçoamento de Pessoal de Nível Superior Brasil (Capes) - Finance Code 001.

\section{References}

1. Belém MD, Ambrosano GM, Tabchoury CP, Ferreira-Santos RI, Haiter-Neto F. Performance of digital radiography with enhancement filters for the diagnosis of proximal caries. Braz Oral Res. 2013 May-Jun;27(3):245-51. https://doi.org/10.1590/S1806-83242013000300004

2. Dashpuntsag O, Yoshida M, Kasai R, Maeda N, Hosoki H, Honda E. Numerical evaluation of image contrast for thicker and thinner objects among current intraoral digital imaging systems. BioMed Res Int. 2017;2017:5215413. https://doi.org/10.1155/2017/5215413

3. Yoshiura K, Nakayama E, Shimizu M, Goto TK, Chikui T, Kawazu T, et al. Effects of the automatic exposure compensation on the proximal caries diagnosis. Dentomaxillofac Radiol. 2005 May;34(3):140-4. https://doi.org/10.1259/dmfr/88681265

4. Hayakawa Y, Farman AG, Scarfe WC, Kuroyanagi K. Processing to achieve high-contrast images with computed dental radiography; technical report. Dentomaxillofac Radiol. 1996 Sep;25(4):211-4. https://doi.org/10.1259/dmfr.25.4.9084276

5. Hayakawa Y, Farman AG, Scarfe WC, Kuroyanagi K. Pixel value modification using RVG-4 automatic exposure compensation for instant high-contrast images. Oral Radiol. 1996;12:11-8. https://doi.org/10.1007/BF02351577

6. Galvão NS, Nascimento EH, Lima CA, Freitas DQ, Haiter-Neto F, Oliveira ML. Can a high-density dental material affect the automatic exposure compensation of digital radiographic images? Dentomaxillofac Radiol. 2019 Mar;48(3):20180331. https://doi.org/10.1259/dmfr.20180331

7. Ismail Al, Sohn W, Tellez M, Amaya A, Sen A, Hasson H, et al. The International Caries Detection and Assessment System (ICDAS): an integrated system for measuring dental caries. Community Dent Oral Epidemiol. 2007 Jun;35(3):170-8. https://doi.org/10.1111/j.1600-0528.2007.00347.x

8. Oliveira ML, Ambrosano GM, Almeida SM, Haiter-Neto F, Tosoni GM. Efficacy of several digital radiographic imaging systems for laboratory determination of endodontic file length. Int Endod J. 2011 May;44(5):469-73. https://doi.org/10.1111/j.1365-2591.2011.01860.x 
Automatic exposure compensation and subjective image enhancement in the radiographic diagnosis of caries

9. Landis JR, Koch GG. The measurement of observer agreement for categorical data. Biometrics. 1977 Mar;33(1):159-74. https://doi.org/10.2307/2529310

10. Nascimento EH, Gaêta-Araujo H, Vasconcelos KF, Freire BB, Oliveira-Santos C, Haiter-Neto F, et al. Influence of brightness and contrast adjustments on the diagnosis of proximal caries lesions. Dentomaxillofac Radiol. 2018 Dec;47(8):20180100. https://doi.org/10.1259/dmfr.20180100

11. Abesi F, Mirshekar A, Moudi E, Seyedmajidi M, Haghanifar S, Haghighat N, et al. Diagnostic accuracy of digital and conventional radiography in the detection of non-cavitated approximal dental caries. Iran J Radiol. 2012 Mar;9(1):17-21. https://doi.org/10.5812/iraniradiol.6747

12. Bottenberg P, Jacquet W, Stachniss V, Wellnitz J, Schulte AG. Detection of cavitated or non-cavitated approximal enamel caries lesions using CMOS and CCD digital X-ray sensors and conventional D and F-speed films at different exposure conditions. Am J Dent. 2011 Apr;24(2):74-8.

13. Dehghani M, Barzegari R, Tabatabai H, Ghanea S. Diagnostic value of conventional and digital radiography for detection of cavitated and noncavitated

14. Kajan ZD, Tayefeh Davalloo R, Tavangar M, Valizade F. The effects of noise reduction, sharpening, enhancement, and image magnification on diagnostic accuracy of a photostimulable phosphor system in the detection of non-cavitated approximal dental caries. Imaging Sci Dent. 2015 Jun;45(2):81-7. https://doi.org/10.5624/isd.2015.45.2.81

15. Krzyżostaniak J, Kulczyk T, Czarnecka B, Surdacka A. A comparative study of the diagnostic accuracy of cone beam computed tomography and intraoral radiographic modalities for the detection of noncavitated caries. Clin Oral Investig. 2015 Apr;19(3):667-72. https://doi.org/10.1007/s00784-014-1282-6

16. Melo SLS, Belem MD, Prieto LT, Tabchoury CP, Haiter-Neto F. Comparison of cone beam computed tomography and digital intraoral radiography performance in the detection of artificially induced recurrent caries-like lesions. Oral Surg Oral Med Oral Pathol Oral Radiol. 2017 Sep;124(3):306-14. https://doi.org/10.1016/i.000o.2017.05.469

17. Soviero VM, Leal SC, Silva RC, Azevedo RB. Validity of MicroCT for in vitro detection of proximal carious lesions in primary molars. J Dent. 2012 Jan;40(1):35-40. https://doi.org/10.1016/i.jdent.2011.09.002

18. Neves AA, Coutinho E, Cardoso MV, Jaecques SV, Van Meerbeek B. Micro-CT based quantitative evaluation of caries excavation. Dent Mater. 2010 Jun;26(6):579-88. https://doi.org/10.1016/j.dental.2010.01.012

19. Boca C, Truyen B, Henin L, Schulte AG, Stachniss V, De Clerck N, et al. Comparison of micro-CT imaging and histology for approximal caries detection. Sci Rep. 2017 Jul;7(1):6680. https://doi.org/10.1038/s41598-017-06735-6

20. Farman AG, Farman TT. A comparison of 18 different x-ray detectors currently used in dentistry. Oral Surg Oral Med Oral Pathol Oral Radiol Endod. 2005 Apr;99(4):485-9. https://doi.org/10.1016/j.tripleo.2004.04.002 